\title{
Computational modeling of calcite cementation in saline limestone aquifers: a phase-field study
}

\author{
Nishant Prajapati ${ }^{*}$ (D), Michael Selzer ${ }^{1,2}$ and Britta Nestler ${ }^{1,2}$
}

*Correspondence: nishant.
prajapati@kit.edu
${ }^{1}$ Institute for Applied
Materials (IAM-CMS),
Karlsruhe Institute
of Technology, Building
30.48, Strasse am Forum 7,
76131 Karlsruhe, Germany
Full list of author information
is available at the end of the
article

Springer Open

\begin{abstract}
The present article investigates the effect of initial grain size on the overall growth kinetics during calcite cementation from supersaturated geothermal fluid whilst tracking the grain boundary behavior of the evolving microstructure using a multiphase-field model. In order to define rhombohedral calcite geometry, we consider a faceted-type surface energy anisotropy and validate the crystal shape using volume preservation technique in three dimensions. Next, we perform calcite growth simulations with multiple grains in 2D as well as 3D in order to computationally mimic the anisotropic cement overgrowths as observed in saline limestone aquifers. A significant deviation in the 3D overgrowth kinetics is observed as compared to 2D. The increase in cement overgrowths is found to be inversely dependent (non-linear) on the initial grain size. Moreover, the grain size distributions obtained from the numerically cemented microstructural data tend to get statistically dispersed and horizontally shifted with increasing mean grain size. Finally, the capability of present modeling approach in simulating dynamics of calcite cementation in $3 \mathrm{D}$ is demonstrated based on postprocessing analyses and advanced visualization techniques.
\end{abstract}

Keywords: Calcite cementation, Multiphase-field model, Overgrowth kinetics, Grain size effect

\section{Background}

Lithification of carbonate sediments in saline environments is a result of complex physical, chemical, and biological processes. During the early periods after deposition, porosities of carbonate muds and sands normally lie in the range of 40-70\% (Pray and Choquette 1966). Most limestones exhibit very little signs of compaction (Pray 1960). Therefore, the reduction of such high porosities to its present low value $(<5 \%)$ is believed to be almost entirely due to introduction of cements (Bathurst 1970). Among a wide spectrum of diagenetic processes that have resulted in the formation of the Devonian fore-reef limestone of Germany or the Silurian limestone of Gotland, Sweden, for instance, cementation has been identified as the major phenomenon that governs the microstructure in carbonate rocks (Krebs 1969; Munnecke et al. 1997). In marine Carboniferous limestone, precipitation of calcium carbonate $\left(\mathrm{CaCO}_{3}\right)$ from saline water results in minerals, filling the interparticle pore spaces. Calcite overgrowth takes place in the same crystallographic orientations as the original sediments and results in faceted

(c) The Author(s) 2017. This article is distributed under the terms of the Creative Commons Attribution 4.0 International License (http://creativecommons.org/licenses/by/4.0/), which permits unrestricted use, distribution, and reproduction in any medium, provided you give appropriate credit to the original author(s) and the source, provide a link to the Creative Commons license, and indicate if changes were made. 
grain boundaries according to rhombohedral crystal morphology (Chafetz et al. 1985). Aragonite, a relatively less stable polymorph of $\mathrm{CaCO}_{3}$, is another rock-forming cement that occurs in limestone (Curl 1962; Sandberg 1985). Due to higher solubility, aragonite dissolves in water and acts as a primary source of $\mathrm{Ca}^{2+}$ and $\mathrm{HCO}_{3}^{-}$, which further reprecipitates as calcite (Folk 1965; Palmer et al. 1988; Frisia et al. 2002).

Carbonate reservoirs account for more than $60 \%$ of oil and $40 \%$ of gas reserves of the world. Therefore, a deep understanding of dynamics of microstructural processes driven by fluid mineral interaction is imperative for the hydrocarbon exploration. There is an extensive literature dealing with quantitative modeling of cementation kinetics based on empirical growth relationships for calcite (Gutjahr et al. 1996; Herwegh and Berger 2003; Rodriguez-Blanco et al. 2011), quartz (Walderhaug 1996), illite (Elliott et al. 1996), and many more. Such studies are advantageous in estimating the petrophysical properties in a quantitative manner. However, they do not provide insights into the processes occurring at micro-scale.

Kinetics of cement overgrowths, among a wide range of hydro-chemical processes, depends primarily on two major factors: the concentration of dissolved $\mathrm{CaCO}_{3}$ in the geothermal fluid and the grain size of aggregates. Teng et al. (2000), Lasaga (2014), Rattas et al. (2014), and Frisia et al. (2015), along with several others, analyzed the role of supersaturation in growth kinetics and proposed empirical relations for the same. Moreover, the grain size dependency of growth rates has been observed for calcite (Kile et al. 2000) as well as other minerals such as dolomite (Nordeng and Sibley 1996), illite (Bove et al. 2002), and quartz (Makowitz and Sibley 2001). The results obtained are as varied as the studies conducted. For instance, Makowitz and Sibley (2001) measured thin sections of natural quartz arenite samples and observed that the quartz overgrowth thickness is directly proportional to the detrital grain radius. Kile et al. (2000) experimentally verified the theoretical models that consider size-dependent growth rates in conjunction with certain nucleation rates which maintain grain size distributions of different shapes.

While hydrothermal flow-through experiments of porous rock samples for laboratory synthesis of cemented microstructures (Ismail et al. 2002) and vein morphologies (Okamoto and Sekine 2011; Bons et al. 2012; McNamara et al. 2016) along with Cathode Luminescence images of thin sections provide a wealth of information about the microstructure of rocks, their limitations in understanding the complex grain boundary interactions during cementation are highly evident. In order to study the diagenetic history and make predictions about the future microstructural evolution, numerical approaches are certainly advantageous. One such study was conducted by Bons (2001) who developed a two-dimensional simulation program called Vein Growth based on front tracking method to explore the mechanism of microstructural developments in fibrous veins. Hilgers et al. (2001) employed the program for studying anisotropic grain growth under different boundary conditions. Lander et al. (2008) separately developed a computational algorithm known as Prism $2 D$ based on continuous value cellular automata approach in order to simulate the dynamic overgrowths in quartz under complex boundary conditions and validated them with experiments. Their numerical results (using Prism2D) as well as observations from the grain growth experiments on quartz seeds of different sizes confirmed the so-called grain size effect-surface area normalized (SAN) growth rate is smaller in fine-grained sandstones than the coarser ones. However, the overall 
cementation rate still tends to be faster in finer grained sandstones. This was attributed to the greater surface area of fine pack surpassing the SAN rate effect. Further, a second outcome of the study is that smaller grains attain euhedral forms faster as compared to the coarser ones (which is also observed in the present study).

Despite the achievements of the above approaches in studying grain growth as well as cementation, their applications have been restricted to two dimensions (2D) owing to enormous computational costs. Grain growth studies in 2D, with lower computational requirements, are expedient in understanding the dynamics of cementation qualitatively. However, there are certain implicit limitations which are often neglected, which are given as follows:

- Complex euhedral geometries of the growing crystals are merely reduced to 2D projections, leading to oversimplification of the problem.

- 2D grain growth simulations are essentially the computational imitation of experimental thin sections of rocks. Ankit et al. (2015) emphasized the role of curvature of grains in the formation of grain boundary patterns in rocks. These curvatures are different in 3D as compared to 2D which might result in erroneous interpretations. Moreover, experimental work of Berger et al. (2011) reported deviations in estimated grain size data of natural rock samples extracted from 2D thin sections (area estimation using image processing tools) in comparison with 3D (using computed tomography, serial sectioning).

Therefore, for a meaningful interpretation of the underlying physics in a realistic manner, the current numerical techniques need to be extended to 3D, whilst assessing the computational efficiency and viability of the modeling approach.

In the present work, we employ a thermodynamically consistent multiphase-field model to simulate the diagenetic cementation over geological time scales (since depositional time to the present day) in calcite-rich limestone aquifers. Phase-field is a diffuse interface approach, used extensively in the materials science community for modeling microstructural evolution during phase transitions (e.g., review articles Chen 2002; Boettinger et al. 2002; Moelans et al. 2008; Nestler and Choudhury 2011). Unlike conventional front tracking methods, phase-field approach obviates the need to track interfaces explicitly, making it a computationally efficient and powerful methodology in treating moving boundary problems such as crack propagation (Miehe et al. 2010; Schneider et al. 2014), fluid flow (Kim et al. 2012), and recently in modeling crack-sealed morphologies in quartz veins (Ankit et al. 2013; Wendler et al. 2015).

The aim of this article is to study the kinetics of calcite overgrowth while gaining insights into grain boundary behavior at micro-scale. At first, we present a systematic study to compare the overgrowth kinetics in $2 \mathrm{D}$ to that in $3 \mathrm{D}$. Next, we address the grain size dependency of the growth rates, whilst tracking the grain boundary interactions in 3D. Finally, we present a brief statistical analysis of the grain size data obtained from numerically cemented microstructures. 


\section{Methods}

\section{Model formulation: multiphase-field model}

In this section, we present a brief description of the multiphase-field model (Nestler et al. 2005) employed to address the phenomenon of calcite cementation in limestone. The model is based on phenomenological minimization of Helmholtz free energy functional formulated as

$$
\mathcal{F}(\boldsymbol{\phi})=\int_{\Omega}\left[f(\boldsymbol{\phi})+\varepsilon a(\boldsymbol{\phi}, \nabla \boldsymbol{\phi})+\frac{1}{\varepsilon} w(\boldsymbol{\phi})\right] \mathrm{d} \Omega,
$$

where the phase-field vector $\boldsymbol{\phi}(\mathbf{x}, \mathrm{t})=\left[\left(\phi_{1}(\mathbf{x}, \mathrm{t}), \ldots, \phi_{N}(\mathbf{x}, \mathrm{t})\right]\right.$ describes the presence of $N$ grains at position $\mathbf{x}$ in space and time $t$, while satisfying the summation constraint $\left(\sum_{\alpha=1}^{N} \phi_{\alpha}=1\right)$ over all phases. Each phase-field parameter $\phi_{\alpha}$ (corresponding to grain $\alpha$ ) varies smoothly from 0 (outside) to 1 (inside the grain), over a diffuse region that characterizes the interfaces. The length scale parameter $\varepsilon$ governs the interfacial width. We discuss each term of the energy functional of Eq. (1) in detail.

The first term is the bulk free energy density which accounts for the interfacial driving forces in the multiphase system. It is formulated as an interpolation of the free energy densities $f_{\alpha}$ of the individual bulk phases

$$
f(\boldsymbol{\phi})=\sum_{\alpha=1}^{N} f_{\alpha} h\left(\phi_{\alpha}\right)
$$

For the present work, we chose an interpolation function $h\left(\phi_{\alpha}\right)=\phi_{\alpha}^{3}\left(6 \phi_{\alpha}^{2}-15 \phi_{\alpha}+10\right)$. In general, any function that satisfies $h(0)=0$ and $h(1)=1$ can be used for interpolation.

The second term represents the gradient energy density which acts as an energetic penalty due to the formation of diffuse interfaces. It is formulated as

$$
a(\boldsymbol{\phi}, \nabla \boldsymbol{\phi})=\sum_{\substack{\alpha, \beta=1 \\(\alpha<\beta)}}^{N, N} \gamma_{\alpha \beta} a_{\alpha \beta}^{2}(\boldsymbol{\phi}, \nabla \boldsymbol{\phi})\left|\mathbf{q}_{\alpha \beta}\right|^{2},
$$

where $\gamma_{\alpha \beta}$ represents the magnitude of surface energy density of the $\alpha-\beta$ interface. The function $a_{\alpha \beta}(\boldsymbol{\phi}, \nabla \boldsymbol{\phi})$ which defines the form of surface energy anisotropy of evolving interfaces is described in "Modeling calcite cements". We use the generalized gradient vector $\mathbf{q}_{\alpha \beta}=\phi_{\alpha} \nabla \phi_{\beta}-\phi_{\beta} \nabla \phi_{\alpha}$ which is normal to the $\alpha-\beta$ interface.

The third term is the multi-obstacle potential energy density. It creates an energy barrier for spontaneous phase transitions in the absence of driving forces. It is expressed as

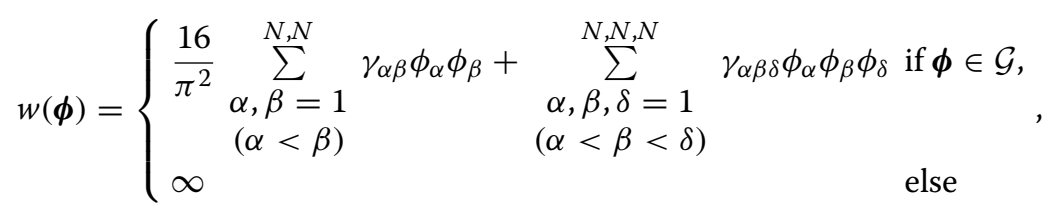

where $\mathcal{G}=\left\{\boldsymbol{\phi} \mid \sum_{\alpha=1}^{N} \phi_{\alpha}=1\right.$ and $\left.\phi_{\alpha} \geq 0\right\}$. The higher order term $\left(\alpha \phi_{\alpha} \phi_{\beta} \phi_{\delta}\right)$ is an artificial construction which prevents the occurrence of unwanted third phases at binary interfaces in a multiphase system. 
Evolution equation of phase-fields is derived from the variational derivative of free energy functional and is given by

$$
\tau \varepsilon \frac{\partial \phi_{\alpha}}{\partial t}=-\frac{\delta \mathcal{F}}{\delta \phi}-\lambda \quad \text { for } \quad \alpha=1, \ldots, N
$$

where $\tau$ denotes the interfacial kinetic coefficient and $\lambda$ is the Lagrange parameter which ensures that the summation constraint $\left(\sum_{\alpha=1}^{N} \phi_{\alpha}=1\right)$ is satisfied. Substituting the expression of free energy functional of Eq. (1) into Eq. (5) yields

$$
\begin{aligned}
\tau \varepsilon \frac{\partial \phi_{\alpha}}{\partial t}= & \varepsilon\left(\nabla \cdot \frac{\partial a(\boldsymbol{\phi}, \nabla \boldsymbol{\phi})}{\partial \nabla \phi_{\alpha}}-\frac{\partial a(\boldsymbol{\phi}, \nabla \boldsymbol{\phi})}{\partial \phi_{\alpha}}\right) \\
& -\frac{1}{\varepsilon} \frac{\partial w(\boldsymbol{\phi})}{\partial \phi_{\alpha}}-\frac{\partial f(\boldsymbol{\phi})}{\partial \phi_{\alpha}}-\lambda
\end{aligned}
$$

with

$$
\begin{aligned}
\lambda= & \frac{1}{N} \sum_{\alpha=1}^{N} \varepsilon\left(\nabla \cdot \frac{\partial a(\boldsymbol{\phi}, \nabla \boldsymbol{\phi})}{\partial \nabla \phi_{\alpha}}-\frac{\partial a(\boldsymbol{\phi}, \nabla \boldsymbol{\phi})}{\partial \phi_{\alpha}}\right) \\
& -\frac{1}{\varepsilon} \frac{\partial w(\boldsymbol{\phi})}{\partial \phi_{\alpha}}-\frac{\partial f(\boldsymbol{\phi})}{\partial \phi_{\alpha}}
\end{aligned}
$$

for $\alpha=1, \ldots, N$.

The partial differential equation in Eq. (6) is solved numerically using forward Euler scheme for the time derivative, while the spatial derivatives are discretized using a second-order accurate central difference scheme. The model equations are implemented in a highly parallelized 3D simulation code, Pace3D 2.2.0 Institute of Materials and Processes (2015) which is written in C language. The code is optimized with the locally reduced order parameter optimization (LROP) that reduces the computational time $\left(O\left(N^{3}\right) \rightarrow O(1)\right)$ and memory consumption $(O(N) \rightarrow O(1))$, with $N$ being the number of phases in the system (Selzer 2014). Such an optimization results in highly efficient computation which is independent of $N$, facilitating 3D large-scale numerical studies. The multiphase-field model presented here has previously been employed by Ankit et al. (2015) for studying microstructural evolution in bitaxial crack seal veins and Wendler et al. (2015) for modeling of epitaxial growth of polycrystalline quartz veins.

\section{Modeling calcite cements}

Amongst a wide spectrum of crystal morphologies, calcite cements have been identified to precipitate in preferred crystallographic orientations primarily forming rhombohedral crystals (Estroff et al. 2004; Kim et al. 2014; Mineral and Gemstone database (2012-2017) 2017); see Fig. 1a. In order to model specific geometries wherein the crystal develops flat facets and sharp edges, we define a strongly anisotropic surface energy by employing the following piecewise function

$$
a_{\alpha \beta}(\boldsymbol{\phi}, \nabla \boldsymbol{\phi})=\max _{1 \leq k \leq n_{\alpha \beta}}\left\{\frac{\mathbf{q}_{\alpha \beta}}{\left|\mathbf{q}_{\alpha \beta}\right|} \cdot \boldsymbol{\eta}_{k, \alpha \beta}\right\},
$$




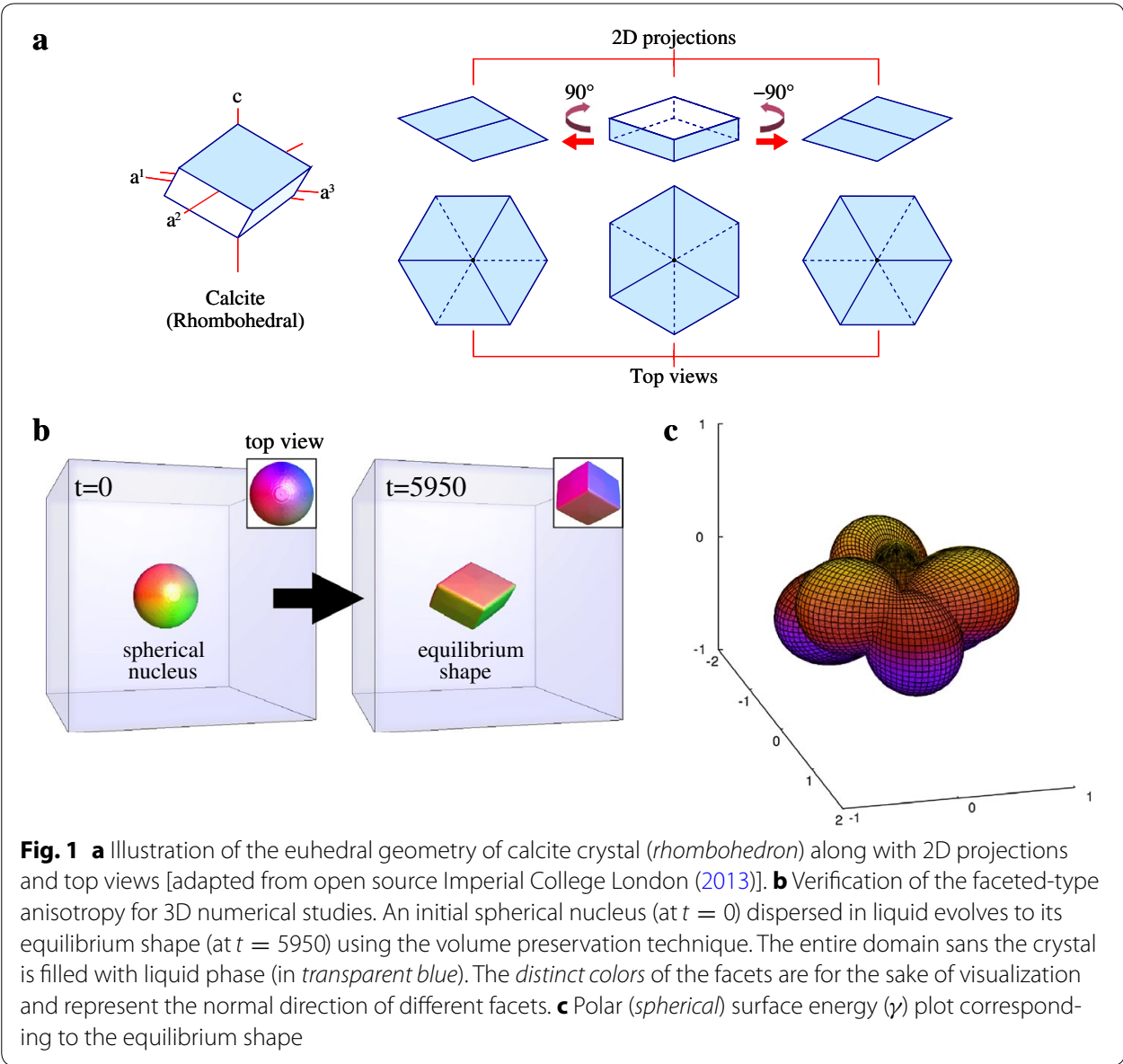

where $\left\{\boldsymbol{\eta}_{k, \alpha \beta} \mid k=1, \ldots, n_{\alpha \beta}\right\}$ are the $n_{\alpha \beta}$ corners of the Wulff shape of the $\alpha-\beta$ transition. This enables us to model any faceted crystal morphology.

In order to validate the faceted-type anisotropic surface energy formulation for equilibrium shape of calcite, we evolve a spherical grain dispersed in liquid to its equilibrium shape while preserving its volume numerically, based on the volume preservation algorithm of Nestler et al. (2008). The initial and final shapes are displayed in Fig. 1b. Figure 1c shows the polar surface energy plots corresponding to the simulated crystal geometry.

\section{Primary assumptions}

For the sake of simplicity, we employ the following assumptions and highlight their relevance in computational modeling of calcite cementation:

1. Carbonate reservoirs are known to be ubiquitously heterogeneous in nature (Westphal 2004; Dou 2011). In the pore spaces of carbonate sediments, there is a wide variety of substrates such as crystalline limestone fragments and carbonate fossils, among others, upon which the nucleation of cement can take place. When the substrate and the cement are the same mineral (eg: calcite), epitaxial overgrowth of cement begins (Bathurst 1972). The resulting events of overgrowth are in optical continuity with 
the original sediments. Moreover, the presence of secondary authigenic minerals as well as clay inhibits the cementation giving rise to nucleation discontinuities (Bloch et al. 2002; Lander et al. 2008; Stricker and Jones 2016). To account for such effects, advanced computational investigations can be conducted by incorporating suitable preprocessing techniques. In this preliminary work, we assume that the substrate is composed entirely of seeds of crystalline calcite grains. Therefore, the precipitation of minerals other than calcite is neglected. Further, the pore space is entirely filled with the geothermal fluid. The influence of inhomogeneity and nucleation discontinuities at pore scale is neglected, for the sake of convenience.

2. During calcite cementation over long ranges, the decrease in supersaturation of the saline geothermal solution is negligible (Bathurst 1972). Therefore, the degree of supersaturation, which is realized by the driving force $f_{\alpha}$ at the grain-liquid interface is assumed to be constant. This essentially implies that the geothermal solution is an infinite reservoir of solute with respect to grain-scale cementation. Having said that, the assumption is applicable in situations where the geothermal fluid is highly supersaturated and viscous, where advection plays a little role (i.e., slowly moving fluids and sluggish kinetics).

3. In the pore space, a grain nucleates into its equilibrium Wulff shape and further grows toward its kinetic Wulff shape, under the action of interfacial processes and long-range transport (Sekerka 2005). The governing mechanism in the evolution of grains is an interplay of surface energy anisotropy and growth kinetics. In the present study, we chose a strong faceted-type anisotropy (Eq. 8) which ensures that nuclei develop flat facets and sharp edges, while growth kinetics is assumed to be isotropic. However, we remark that the anisotropy in the growth kinetics can be easily accounted by employing similar piecewise function in the interfacial kinetic coefficient $\tau$ and is not a limitation of the phase-field approach, in general (Nestler et al. 2005; Wendler et al. 2015).

4. In the present work, we focus on calcite mineralization under isothermal conditions at temperatures which are sufficiently below the recrystallization temperature. Under such thermal conditions, the grain boundaries behave in a rigid manner. Therefore, the kinetic coefficient for grain-grain interaction has been assigned sufficiently high values in comparison to grain-liquid interaction in order to ensure rigid grain-grain interfaces. However, the effects arising due to thermal gradients and temporally evolving temperatures can be included by incorporating temperature dependencies in the driving force $f_{\alpha}$, interfacial kinetic coefficient $\tau$, and surface energy density $\gamma_{\alpha \beta}$ of the $\alpha-\beta$ interface. Furthermore, the phase-field equation (in Eq. 6) should be coupled with an additional temperature evolution equation.

\section{Results and discussion}

Growth in 2D versus 3D

Numerical studies in 2D cannot be compared to the 3D counterparts in a straightforward manner, due to the additional degree of freedom of the evolving grains in 3D. For a meaningful comparison, the following preprocessing procedure has been employed: 
- Generation of $3 D$ computational domain: in a cubic domain of size $300 \Delta x \times 300 \Delta x \times 300 \Delta x$ (where $\Delta x$ denotes the grid size of the finite difference solver), a distribution of spherical grains is generated using a Distribution generating algorithm. The algorithm generates random numbers for the coordinates of the center of each grain (of constant grain size $r=20 \Delta x$ ), while filtering out grains which overlap the already generated ones. The procedure continues introducing new grains within the domain until the probability of inserting an additional grain without overlap goes to zero. The resulting distribution consists of grains which are touching (or almost touching) one or several neighboring grains; see Fig. 2a. Further, using a random number generator, each grain in the 3D computational domain is assigned a random crystallographic orientation defined by Euler angle rotations $\left(\theta_{1}, \theta_{2}, \theta_{3}\right)$; see Fig. 3a.

- Generation of $2 D$ computational domain: a central thin section of thickness $3 \Delta x$ is extracted from the generated $3 \mathrm{D}$ domain in order to obtain the $2 \mathrm{D}$ computational domain, as shown in Fig. 2b. The crystallographic orientation of each grain in the central thin section is assigned the same value as that of the grains in the central plane of 3D domain.

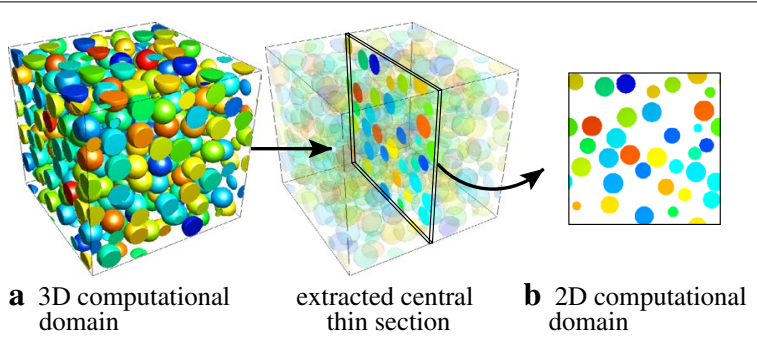

Fig. 2 Initial fillings of 2D and 3D computational domains. a 3D seeds of grains resulting from the distribution generating algorithm. b 2D computational domain obtained by extracting a thin (central) section of the 3D domain

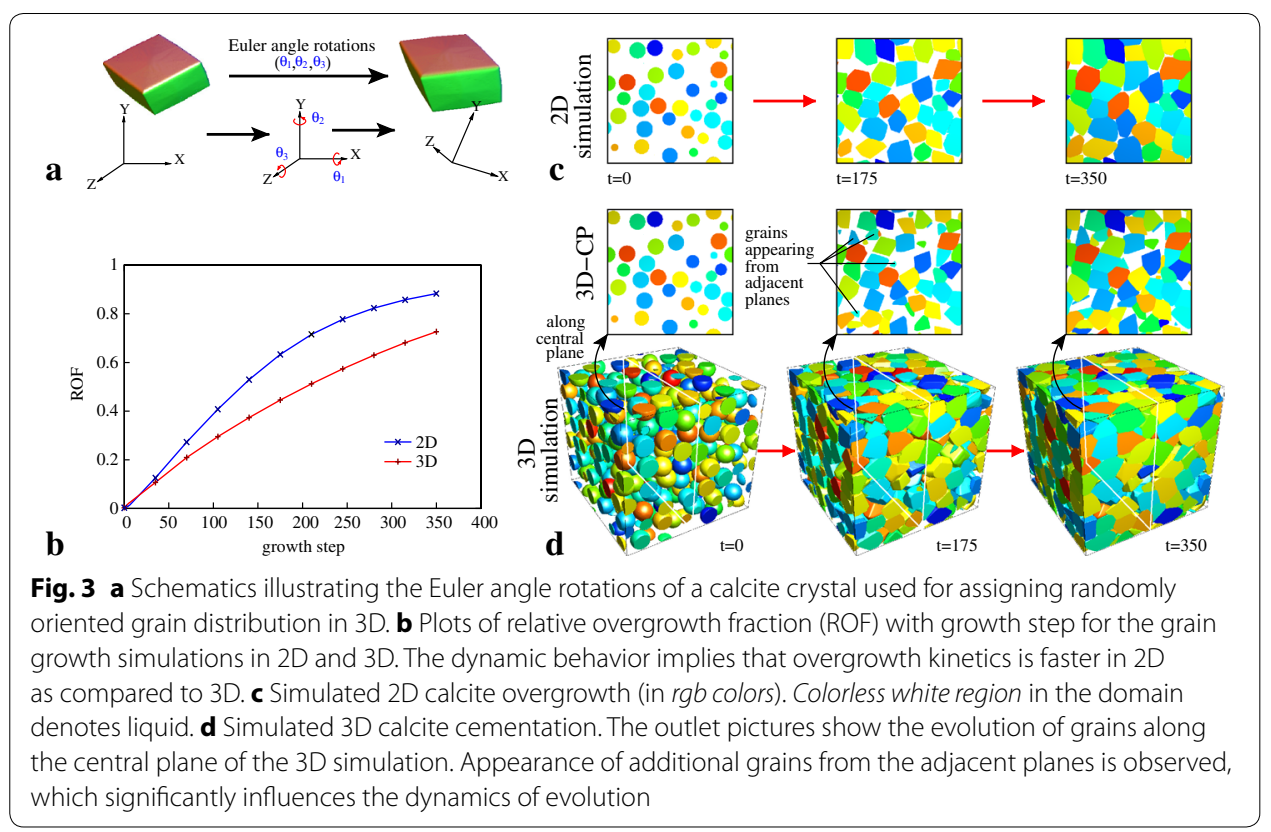


- A constant driving force $f_{\alpha}$ is applied for both setups. For the 2D case, the driving force is scaled appropriately to ensure equivalence with the 3D setup, based on fundamentals of phase-field (Plapp 2012).

- Periodic boundary conditions are applied in all directions of 2D and 3D computational domains.

With the above setups, phase-field simulations were performed. We introduce a measure, relative overgrowth fraction (ROF), defined as

$$
\mathrm{ROF}=\frac{f l_{0}-f l_{t}}{f l_{0}}=\frac{f_{o g}}{f l_{0}},
$$

where $f l_{0}$ and $f l_{t}$ denote the volume fraction of liquid at growth step $t_{0}(=0)$ and $t$, respectively. ROF is the measure of the amount of overgrowth with respect to the initial amount of liquid present in the system. The difference $f l_{0}-f l_{t}$ is equal to the volume fraction of overgrowths $f_{o g}$. With every growth step, a decrease in the volume fraction of liquid $f l_{t}$ takes place due to overgrowth of calcite grains. Irrespective of the domain size and dimension, ROF tends toward 1 as the microstructure is sealed completely due to cementation. We plot the temporal evolution of ROF for 2D and 3D; see Fig. 3b. A significant difference between the overgrowth kinetics of $2 \mathrm{D}$ and $3 \mathrm{D}$ is observed. The plot essentially implies that grain growth kinetics is much faster in 2D as compared to 3D. Figure 3c, d depicts the different stages of growth of calcite grains in $2 \mathrm{D}$ and 3D, respectively. We compare the evolution of grains along the central plane of the 3D setup (3D-CP) with the 2D simulation (in which the initial grain distribution is identical to that of 3D-CP). As the grain growth proceeds, in 3D, grains from adjacent planes penetrate into the central plane and start influencing the dynamics of inplane evolution. This can be clearly seen in Fig. 3c, $\mathrm{d}$ at growth step $t=175$. At $t=350$, we observe that the amount of overgrowth is significantly higher in $2 \mathrm{D}$ setup as compared to that in $3 \mathrm{D}-\mathrm{CP}$. Therefore, the observed reduced rate of overgrowth in 3D can safely be attributed to the higher number of grain boundary interactions.

\section{Influence of initial grain size}

In this section, we investigate the effect of initial grain size $r$ on overgrowth kinetics in 3D.

\section{Model test case: unconstrained monograin growth}

As a model test case, we simulated the unconstrained growth of a monograin in a cubic domain filled with liquid for three different grain sizes. For the sake of convenience, we refer to them as fine, medium, and coarse grains. The domain size for the three monograins (as shown in Table 1) is such that the initial volume fraction (at $t=0$ ) of grain and liquid in each case is identical. Figure $4 \mathrm{a}-\mathrm{c}$ illustrates the temporally evolving isosurfaces of fine, medium, and coarse monograins, respectively. The colormap of isosurfaces (except at $t=0)$ corresponds to the curvature $\kappa=\nabla \cdot\left(\mathbf{q}_{\alpha \beta} /\left|\mathbf{q}_{\alpha \beta}\right|\right)$. As the unconstrained growth proceeds, we observe the development of facets and edges in monograins at $t=175$. However, the shape of fine grain with sharper corner (in red) is closer to its 
Table 1 Simulation domains corresponding to the initial grain size $r$ of monograin for unconstrained growth

\begin{tabular}{lll}
\hline Monograin & Grain size $r$ & Simulation domain size \\
\hline Fine & $20 \Delta x$ & $200 \Delta x \times 200 \Delta x \times 200 \Delta x$ \\
Medium & $25 \Delta x$ & $250 \Delta x \times 250 \Delta x \times 250 \Delta x$ \\
Coarse & $30 \Delta x$ & $300 \Delta x \times 300 \Delta x \times 300 \Delta x$ \\
\hline
\end{tabular}

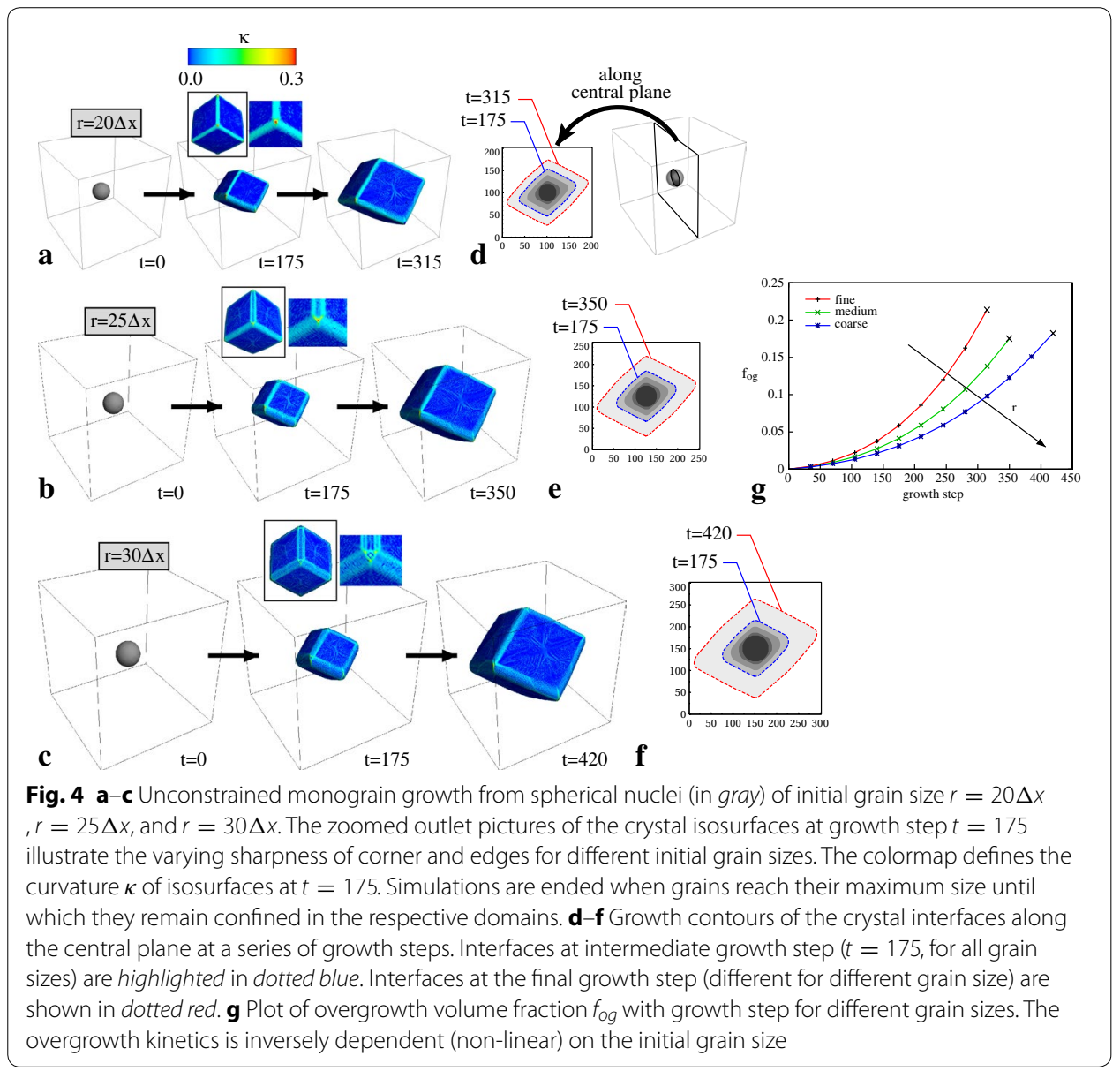

euhedral shape, while the corners of medium and coarse grains are still blunt (bluish red region). Further, the edges (thin sky bluish region) of fine grain are closer to being sharp than the medium and coarse grains. This essentially implies that finer grains attain their euhedral form faster compared to the coarser ones. This has also been observed in experiments concerning quartz cementation (Lander et al. 2008) and is explained by the authors as follows: "overgrowths that nucleate on larger sites must grow farther before they reach euhedral termination." For larger grains, a higher number of surface layers have to be appended in order to attain the final equilibrium shape. Assuming that the time taken for addition of each new layer is independent of the grain size, it clearly follows that larger grains attain equilibrium slower than the fine ones. This also holds in the phase-field approach where the activity of phase changes is concentrated only 
at the interfaces. Moreover, in the current model, the driving forces that induce shape changes increase with curvature. Therefore, the fine grain attains its equilibrium shape faster than the coarser ones, as observed in Fig. 4a-c. Figure $4 \mathrm{~g}$ comprises the evolution of unconstrained overgrowths as a function of growth steps. We deduce that the rate of overgrowth volume fraction is higher for fine grains and decreases as the grain size increases. Figure $4 \mathrm{~d}-\mathrm{f}$ shows the temporal evolution of the isosurfaces along the central plane of different sized monograins. The smaller the size of monograin, the lesser the time required to reach its maximum size, i.e., just before it touches the periodic edges of the domain. The difference between the behavior exhibited by the three cases considered is due to the curvatures involved at the beginning of the simulation. On scaling the governing Eq. (6) using domain width as a characteristic length scale for each of the three cases, it can be seen that the only difference occurs in the term that corresponds to the gradient energy density term (first parenthesis of the RHS of Eq. 6), the difference being the factoring out of square of the reciprocal of the domain width (which is proportional to the curvature). Although the exact same explanation (i.e., scaling argument) does not apply for the later stages of the simulation, the difference can still safely be attributed to the curvatures which are always higher for smaller grains.

\section{Multigrain system}

In order to capture the effect of initial grain size, three scaled distributions were generated in proportion to the grain sizes using the distribution generating algorithm (described in "Growth in 2D versus 3D"). In order to maintain an identical relative configuration of grains for different grain sizes, the coordinates of grains were scaled in proportion to the initial grain size. That is, for a position $\left(x_{1}, x_{2}, x_{3}\right)$ of a grain of size $r \Delta x$ in a cubic domain of edge $n \Delta x$, the position of the corresponding grain of size $2 r \Delta x$ in a scaled cubic domain of edge $2 n \Delta x$ is $\left(2 x_{1}, 2 x_{2}, 2 x_{3}\right)$. Such an initial setting ensures that the effect arising due to neighboring grain interactions is identical. Furthermore, it also guarantees an equal initial volume fraction of grains $(\approx 33 \%)$ in the numerical domain for different grain sizes. In naturally occurring near-surface carbonate rocks, the effect of compaction is negligible, giving rise to high porosities. As the depth increases, the porosity decreases exponentially (Halley and Schmoker 1983). During early periods after deposition, primary porosity of uncompacted carbonate rocks ranges from 40 to $70 \%$ (Pray and Choquette 1966). The present work deals with calcite mineralization in nearsurface uncompacted limestones. Therefore, a high initial porosity $(\approx 67 \%)$ in the computational domain seems reasonable. The domain sizes for multigrain simulations are shown in Table 2. With a constant driving force $f_{\alpha}(=-0.3)$, grain growth simulations were performed for different grain sizes. We plot the overgrowth volume fraction $f_{o g}$

Table 2 Simulation domains corresponding to the initial grain size $r$ for multigrain simulations

\begin{tabular}{lll}
\hline Grains & Grain size $r$ & Simulation domain size \\
\hline Fine & $20 \Delta x$ & $300 \Delta x \times 300 \Delta x \times 300 \Delta x$ \\
Medium & $25 \Delta x$ & $375 \Delta x \times 375 \Delta x \times 375 \Delta x$ \\
Coarse & $30 \Delta x$ & $450 \Delta x \times 450 \Delta x \times 450 \Delta x$ \\
\hline
\end{tabular}




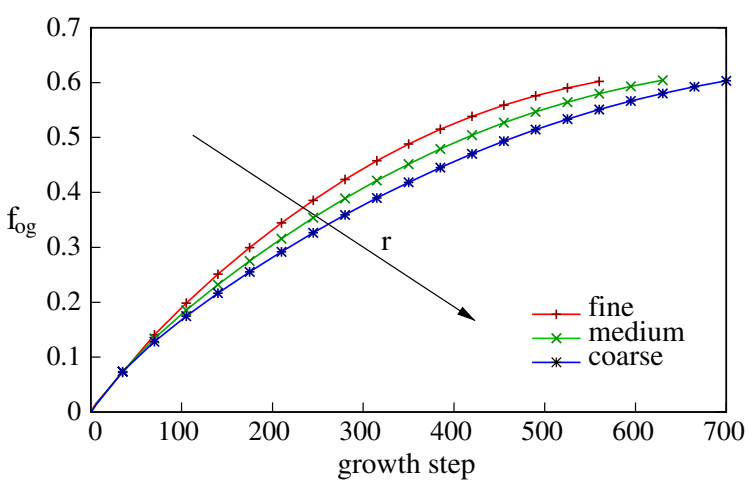

Fig. 5 Plots of overgrowth volume fraction $f_{o g}$ with growth step for fine, medium, and coarse grains. Overgrowth kinetics is inversely dependent (non-linear) on initial grain size

with growth steps; see Fig. 5. The rate of overgrowth is observed to be the highest for fine grains and decreases as the initial grain size increases. This essentially implies that overgrowth kinetics is inversely dependent (non-linear) on the initial grain size. We refer this as grain size effect. The overgrowth trends in Fig. 5 can be rationalized on the arguments provided for the unconstrained growth. The additional complexities arising due to the grain boundary interactions do not affect the mechanism based on curvatures. Therefore, although the kinetics may differ, the principal behavior of the overgrowth curve for finer grains lying over the coarser ones remains the same. Similar experimental studies were conducted by Lander et al. (2008) (with quartz grains), who reported the fastest rate of cementation in fine-grained system. Figure $6 \mathrm{a}-\mathrm{c}$ depicts the different sized multigrain systems comprising calcite grains and liquid at $t=0$. In order to gain insights

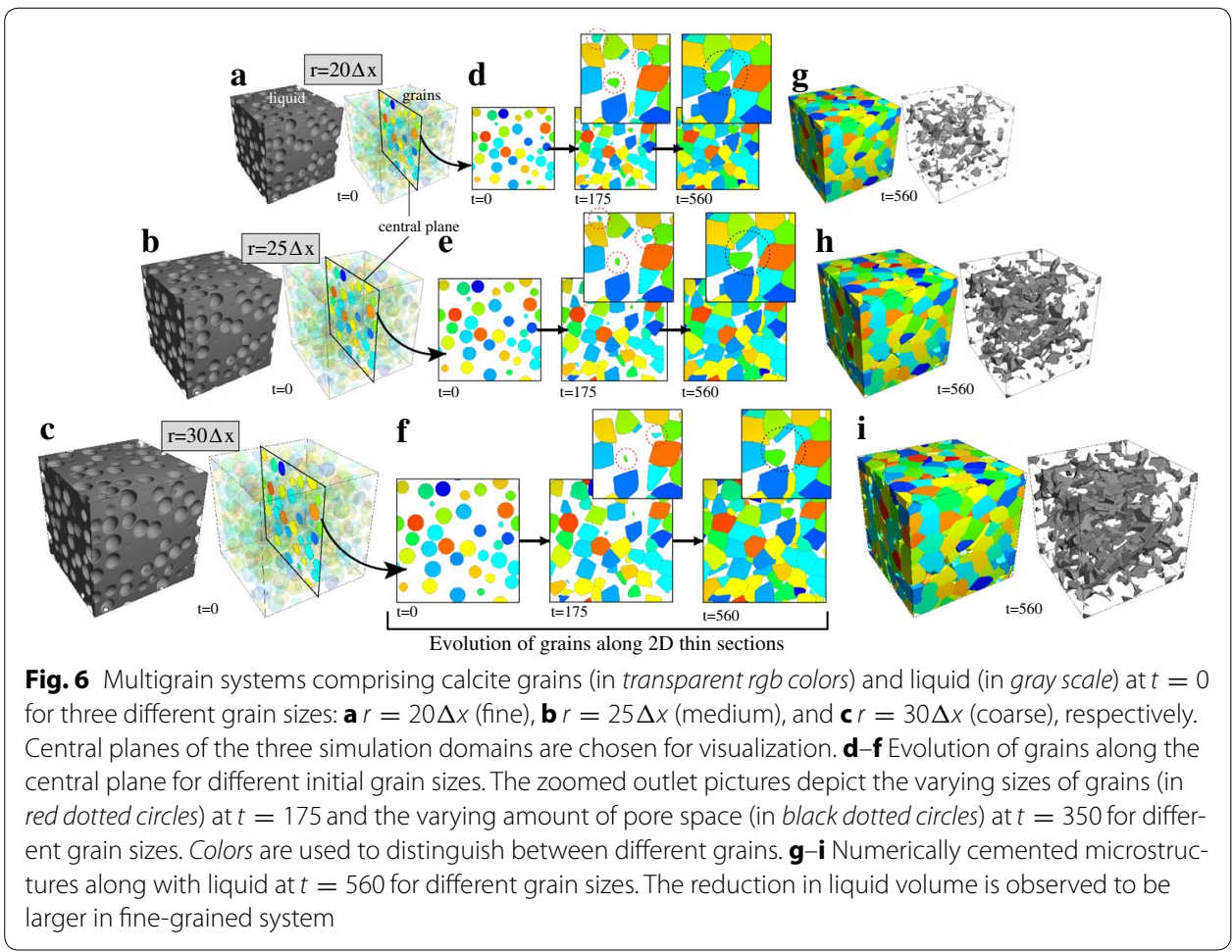


into the microstructural processes, we visualize the evolution of grains along the central plane for the three grain sizes. Figure $6 \mathrm{~d}-\mathrm{f}$ illustrates the different stages of the simulated calcite cementation along the central plane for fine, medium, and coarse grains, respectively. As the growth proceeds, the following characteristic differences are observed: At $t=0$, the grain distributions for all grain sizes are identical. At $t=175$, the amount of growth of grains is larger in fine multigrain systems (as highlighted by the dotted red circles) and decreases with initial grain size. As a result, the amount of pore space sealing is more pronounced for fine-grained systems compared to the coarser ones. This effect can be seen in Fig. $6 \mathrm{~d}-\mathrm{f}$ at $t=560$. Figure $6 \mathrm{~g}-\mathrm{i}$ shows the numerically cemented microstructures (in rgb colors) and the remaining liquid (in gray scale) at $t=560$. It can be visually observed that the reduction in liquid due to overgrowths is larger in fine-grained system. The present study predicts the grain size effect during calcite cementation, qualitatively. However, in order to comment on the grain statistics of the cemented microstructure, the number of grains is certainly low ( $=265$ nuclei). Statistical data extracted from natural samples of calcite-cemented microstructures (Kile et al. 2000) follow a lognormal grain size distribution (GSD). Further, they report a statistical dispersion and horizontal shift in the GSD with increasing mean grain size.

We plot the GSD histograms of the numerically cemented microstructures when porosities are low $(5-7 \%)$ and sufficiently high volume fraction of overgrowths $(\approx 0.6)$ have taken place for all three grain sizes; see Fig. 7. The observed characteristics in the GSDs are as follows:

- From the GSD histograms, mean grain sizes of $28.8 \Delta x, 35.8 \Delta x$, and $42.9 \Delta x$ are obtained corresponding to the initial grain sizes $r=20 \Delta x, 25 \Delta x$, and $30 \Delta x$, respectively.

- For fine grains, GSD is sharp. As the mean grain size increases, GSD gets more dispersed leading to lowering in the peak. This essentially implies that the growth rates of individual fine grains are not as varied as those of the coarser ones, resulting in the sharper GSD. The reason for the lowering of peaks for coarser grains directly follows from this due to the equal number of grains for the three cases.

- As the mean grain size increases, a horizontal shift in the peaks with respect to the initial grain size is observed.

The above-mentioned observations are in qualitative agreement with natural examples reported in Fig. 9 of Kile et al. (2000). However, the lognormal distribution is not well predicted in the present numerical study, owing to the small number of grains.

\section{Conclusion and outlook}

In the present study, the effect of initial grain size on the kinetics of calcite cementation is studied using a multiphase-field model. The comparison of simulation results of 2D with 3D indicates a significant difference in the predicted overgrowth kinetics. 3D simulations for different grain sizes propose an inverse relation (non-linear) between overgrowth kinetics and initial grain size. Unconstrained calcite growth simulations show that the rate of unconstrained overgrowths is also inversely related to the initial grain size. Further, it is observed that fine grains attain euhedral shape more rapidly in comparison 

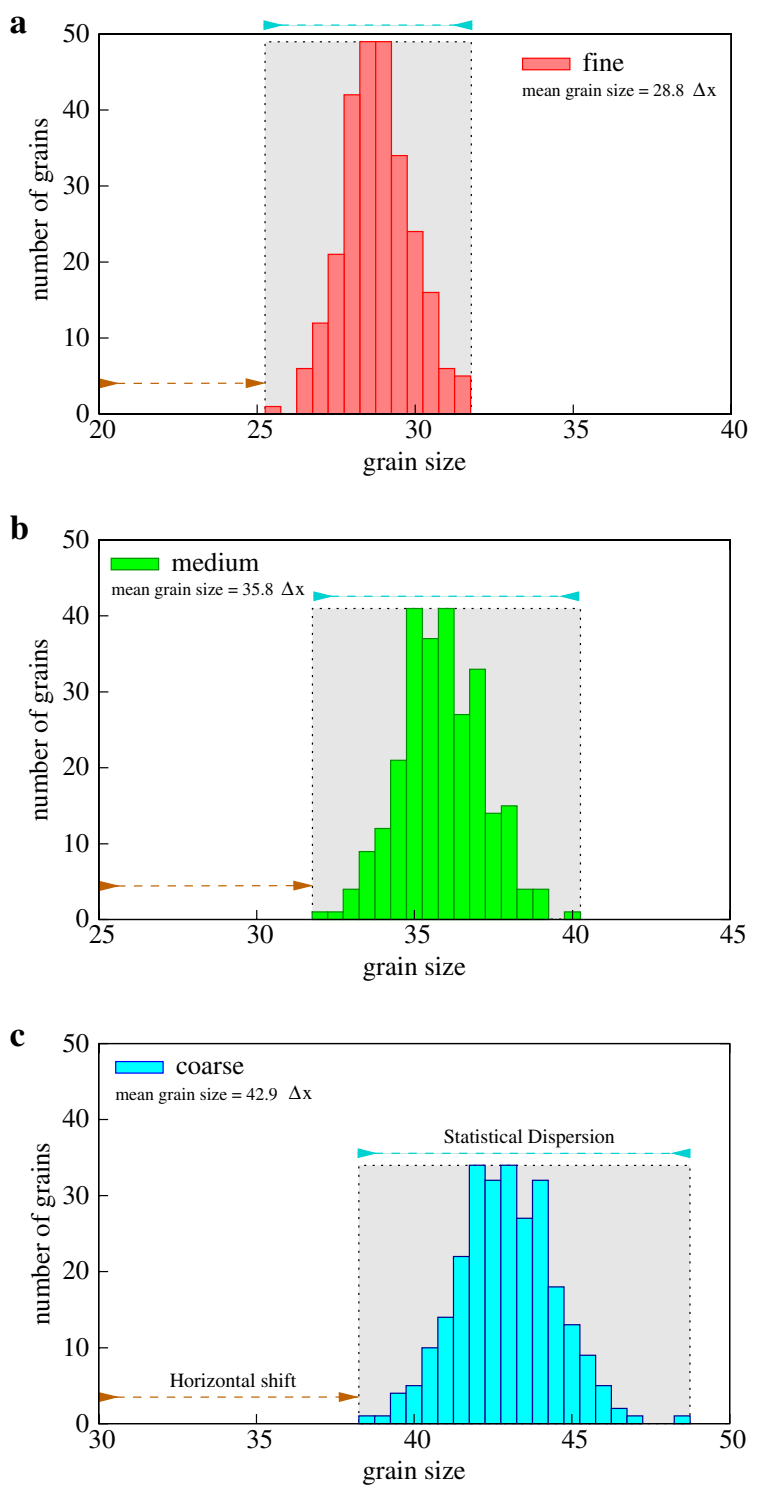

Fig. 7 Histogram plots of grain size distribution of the numerically cemented microstructure corresponding to the grain sizes: $\mathbf{a} r=20 \Delta x, \mathbf{b} r=25 \Delta x$, and $\mathbf{c} r=30 \Delta x$, respectively. The dotted boxed region (in gray) is for visual illustration of horizontal shift and dispersion in the GSD with an increase in mean grain size

to the coarser ones. Similar grain growth studies conducted by Lander et al. (2008) for quartz report this effect owing to the euhedral termination. It is noteworthy that the present multiphase-field model which includes faceted surface energy anisotropy, a constant driving force between the grain-liquid interfaces and isotropic growth kinetics, is successful in capturing grain size effect in a qualitative manner. Next, we compared the GSDs of the numerically cemented microstructure of fine, medium, and coarse grains. The GSDs exhibit a statistical dispersion and horizontal shift with increasing mean grain size. These characteristics of the grain size data are in qualitative agreement with the 
experimental work of Kile et al. (2000). The statistical data obtained from natural samples predict a lognormal distribution of calcite grains. Due to the inadequate number of grains for comprehensive statistical analysis, the predicted distribution is not visibly lognormal. Clearly, the present study is far from being complete. For a rigorous analysis of grain statistics, large-scale numerical studies need to be conducted that can further be compared to natural samples. The present work, whilst providing valuable impressions of the influence of initial grain size on calcite overgrowth kinetics, falls short of the complete treatment of the complicated and rich phenomenon of cementation. One of the immediate steps toward a more complete treatment would be incorporation of diffusional processes that mediate the process of cementation. The model could be further extended to investigate the influence of temperature gradients. Moreover, incorporating appropriate kinetic anisotropy in the formulation would facilitate the calibration of overall overgrowth kinetics and validation with benchmark cementation experiments. It might also be interesting to investigate the competitive growth of aragonite and calcite, when both are present as substrate (Bathurst 1972). This could provide further insights into the mechanism of formation of competitive cement growth fabrics where several mineral substrates are present.

\section{Authors' contributions}

NP conducted the numerical studies under the supervision of MS in addition to conceptual discussions with BN. NP analyzed the numerical results with the help of MS and drafted the manuscript. All authors edited the manuscript. All authors read and approved the final manuscript.

\section{Author details}

${ }^{1}$ Institute for Applied Materials (IAM-CMS), Karlsruhe Institute of Technology, Building 30.48, Strasse am Forum 7 ,

76131 Karlsruhe, Germany. ${ }^{2}$ Institute of Materials and Processes (IMP), Karlsruhe University of Applied Sciences, Moltkestr. 30, 76133 Karlsruhe, Germany.

\section{Acknowledgements}

The authors would like to thank the Helmholtz Association for the financial support through the program "EMR-Energy efficiency, Materials and Resources" and "KIT- Geothermal integration initiative" within the program "RE-renewable energies." Further, they acknowledge publication support by Helmholtz Centre for Environmental Research-UFZ; Helmholtz Centre Potsdam-GFZ German Research Centre for Geosciences, and Karlsruhe Institute of Technology for the article processing charges.

\section{Competing interests}

The authors declare that they have no competing interests.

\section{Availability of data and materials}

All relevant data and material are presented in the main paper.

\section{Consent for publication}

Not applicable.

Ethics approval and consent to participate

Not applicable.

\section{Appendix}

As previously discussed, the present phase-field model provides a general framework to simulate crystals of any faceted geometry. Exemplarily, we consider the simulated crystal geometry of orthorhombic aragonite shown in Fig. 8, which can be used for further numerical studies. 

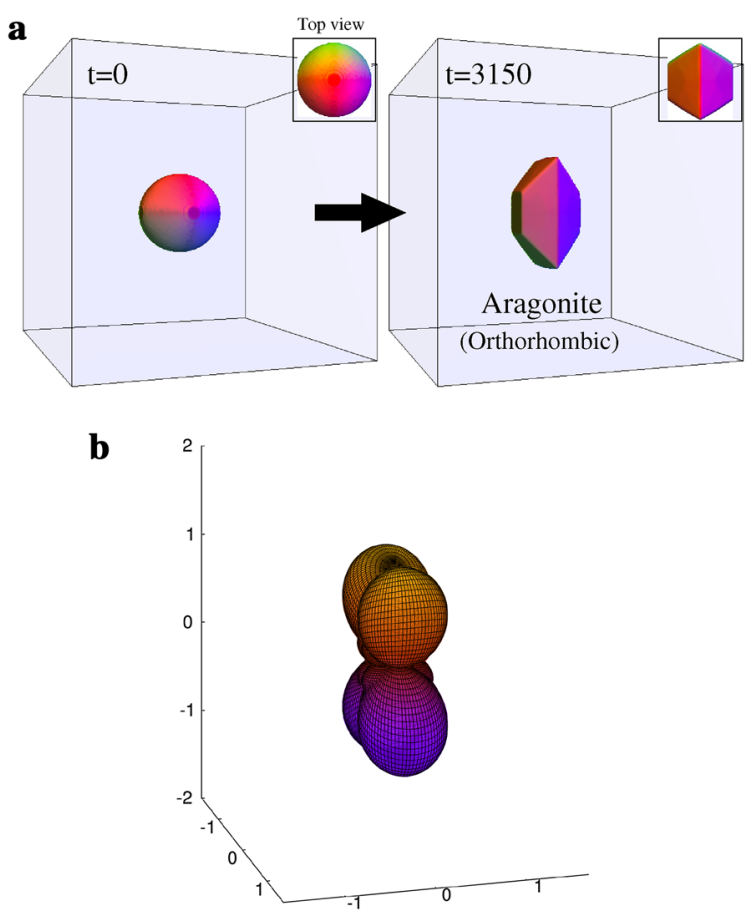

Fig. 8 a Simulated aragonite geometry using the volume preservation technique [crystal shape adapted from open source Imperial College London (2013)]. b Polar (spherical) surface energy ( $\gamma$ ) plot corresponding to the equilibrium shape of the aragonite crystal in $3 \mathrm{D}$

\section{Publisher's Note}

Springer Nature remains neutral with regard to jurisdictional claims in published maps and institutional affiliations.

Received: 3 April 2017 Accepted: 2 August 2017

Published online: 12 August 2017

\section{References}

Ankit K, Nestler B, Selzer M, Reichardt M. Phase-field study of grain boundary tracking behavior in crack-seal microstructures. Contrib Miner Petrol. 2013;166(6):1709.

Ankit K, Urai JL, Nestler B. Microstructural evolution in bitaxial crack-seal veins: a phase-field study. J Geophys Res Solid Earth. 2015:120(5):3096-118.

Antonio LC, et al. Kinetic theory in the earth sciences. New Jersey: Princeton University Press; 2014

Bathurst RGC. Problems of lithification in carbonate muds. Proc Geolog Assoc. 1970;81(3):429-40.

Berger A, Herwegh M, Schwarz JO, Putlitz B. Quantitative analysis of crystal/grain sizes and their distributions in 2D and 3D. J Struc Geol. 2011;33(12):1751-63.

Bloch S, Lander RH, Bonnell L. Anomalously high porosity and permeability in deeply buried sandstone reservoirs: Origin and predictability. AAPG Bull. 2002;86(2):301-28.

Boettinger WJ, Warren JA, Beckermann C, Karma A. Phase-field simulation of solidification. Annu Rev Mater Res. 2002;32(1):163.

Bons P. Development of crystal morphology during unitaxial growth in a progressively widening vein: I. the numerical model. J Struc Geol. 2001;23(6):865-72.

Bons PD, Elburg MA, Gomez-Rivas E. A review of the formation of tectonic veins and their microstructures. J Struc Geol. 2012:43:33-62.

Bove D, Eberl D, McCarty D, Meeker G. Characterization and modeling of illite crystal particles and growth mechanisms in a zoned hydrothermal deposit, Lake City, Colorado. Am Mineral. 2002;87(11-12):1546-56.

Chafetz HS, Wilkinson BH, Love KM. Morphology and composition of non-marine carbonate cements in near-surface settings. 1985

Chen LQ. Phase-field models for microstructure evolution. Annu Rev Mater Res. 2002;32(1):113-40

Curl RL. The aragonite-calcite problem. Bull Natl Speleol Soc. 1962;24(1):57-73.

Dou Q, Sun Y, Sullivan C. Rock-physics-based carbonate pore type characterization and reservoir permeability heterogeneity evaluation, Upper San Andres reservoir, Permian Basin, west Texas. J Appl Geophys. 2011;74(1):8-18. 
Elliott WC, Matisoff G. Clays Clay Miner. 1996;44(1):77-87.

Estroff LA, Incarvito CD, Hamilton AD. Design of a synthetic foldamer that modifies the growth of calcite crystals. J Am Chem Soc. 2004;126(1):2-3.

Frisia S. Aragonite-calcite relationships in speleothems (Grotte de Clamouse, France): environment, fabrics, and carbonate geochemistry. J Sedim Res. 2002;72(5):687.

Frisia S. Microstratigraphic logging of calcite fabrics in speleothems as tool for palaeoclimate studies. Int J Speleol. 2015;44(1):1.

gem5. Mineral and gemstone database (2012-2017). gem5.com (2012-2017)

Gutjahr A, Dabringhaus H, Lacmann R. Studies of the growth and dissolution kinetics of the CaCO3 polymorphs calcite and aragonite I. Growth and dissolution rates in water. J Cryst Growth. 1996;158(3):296.

Halley RB, Schmoker JW. High-porosity Cenozoic carbonate rocks of south Florida: progressive loss of porosity with depth. AAPG Bull. 1983;67(2):191-200.

Herwegh M, Berger A. Differences in grain growth of calcite: a field-based modeling approach. Contrib Miner Petrol. 2003;145(5):600-11.

Hilgers C, Koehn D, Bons P, Urai J. Development of crystal morphology during unitaxial growth in a progressively widening vein: II. Numerical simulations of the evolution of antitaxial fibrous veins. J Struc Geol. 2001;23(6):873-85.

Imperial College London. Imperial college rock library (2013). https://wwwf.imperial.ac.uk/. 2013.

Institute of Materials and Processes, Karlsruhe University of Applied Sciences, Molkestr. 30, 76133 Karlsruhe. Parallel algorithm for crystal evolution in 3-d. 2015. https://www.hs-karlsruhe.de/forschung/imp/cmse/pace-3d-software

Ismail M, Joer H, Randolph M, Meritt A. Cementation of porous materials using calcite. Geotechnique. 2002;52(5):5.

Kile D, Eberl D, Hoch A, Reddy M. An assessment of calcite crystal growth mechanisms based on crystal size distributions. Geochimica et Cosmochimica Acta. 2000;64(17):2937-50

Kim J. Phase-field models for multi-component fluid flows. Commun Comput Phys. 2012;12(03):613-61.

Krebs W. Early void-filling cementation in Devonian fore-reef limestones (Germany). Sedimentology. 1969;12(3--4):279-99.

Lander RH, Larese RE, Bonnell LM. Toward more accurate quartz cement models: the importance of euhedral versus noneuhedral growth rates. AAPG Bull. 2008;92(11):1537.

Makowitz A, Sibley D. Crystal growth mechanisms of quartz overgrowths in a Cambrian quartz arenite. J Sedim Res. 2001;71(5):809-16.

McNamara DD, Lister A, Prior DJ. Calcite sealing in a fractured geothermal reservoir: insights from combined EBSD and chemistry mapping. JVolcanol Geotherm Res. 2016;323:38-52.

Miehe C, Welschinger F, Hofacker M. J Mech Phys Solids. 2010;58(10):1716-40.

Moelans N, Blanpain B, Wollants P. An introduction to phase-field modeling of microstructure evolution. Calphad. 2008;32(2):268-94.

Munnecke A. Microspar development during early marine burial diagenesis: a comparison of Pliocene carbonates from the Bahamas with Silurian limestones from Gotland (Sweden). Sedimentology. 1997;44(6):977-90.

Nestler B, Choudhury A. Phase-field modeling of multi-component systems. Curr Opin Solid State Mater Sci. 2011;15(3):93-105.

Nestler B, Garcke H, Stinner B. Multicomponent alloy solidification: phase-field modeling and simulations. Phys Rev. 2005;71(4):041609.

Nestler B, Wendler F, Selzer M, Stinner B, Garcke H. Phase-field model for multiphase systems with preserved volume fractions. Phys Rev. 2008;78(1):011604

Nordeng SH, Sibley DF. A crystal growth rate equation for ancient dolomites: evidence for millimeter-scale flux-limited growth. J Sedim Res. 1996;66:3.

Okamoto A, Sekine K. Textures of syntaxial quartz veins synthesized by hydrothermal experiments. J Struc Geol. 2011:33(12):1764-75.

Palmer TJ, Hudson J, Wilson MA. Palaeoecological evidence for early aragonite dissolution in ancient calcite seas. Nature. 1988;335(6193):809-10

Plapp P. Mathis. Phase-field models. multiphase microfluidics: the diffuse interface model. 2012. p. 129

Pray LC. Compaction in calcilutites. Bull Geol Soc Am. 1960;71(122):1946.

Pray LC, Choquette PW. Genesis of carbonate reservoir facies. AAPG Bull. 1966;50(3):632.

Rattas M, Lomp P, Jõeleht A. Carbonate cementation in the late glacial outwash and beach deposits in northern Estonia. Estonian J Earth Sci. 2014;63(1):30

Robert FL. Some aspects of recrystallization in ancient limestones. Dolomitization Limest Diagenesis. 2010;1 (1):14.

Robin GC. Carbonate sediments and their diagenesis. Vol. 12, Elsevier, 1972

Rodriguez-Blanco JD, Shaw S, Benning LG. The kinetics and mechanisms of amorphous calcium carbonate (ACC) crystallization to calcite, via vaterite. Nanoscale. 2011:3(1):265-71.

Sandberg P. Aragonite cements and their occurrence in ancient limestones. 1985.

Schneider D, Selzer M, Bette J, Rementeria I, Vondrous A, Hoffmann MJ, Nestler B. Phase-field modeling of diffusion coupled crack propagation processes. Adv Eng Mater. 2014;16(2):142.

Sekerka RF. Equilibrium and growth shapes of crystals: how do they differ and why should we care? Cryst Res Technol. 2005:40(4-5):291-306.

Selzer M. Mechanische und strömungsmechanische topologieoptimierung mit der phasenfeldmethode. Ph.D. thesis, Ph. D. thesis, Department of Mechanical Engineering., Karlsruhe Institute of Technology, Germany; 2014.

Stephan S, Jones SJ. Enhanced porosity preservation by pore fluid overpressure and chlorite grain coatings in the Triassic Skagerrak, Central Graben, North Sea, UK. London: Special Publications;2016. p. SP435.

Teng HH, Dove PM, De Yoreo JJ. Kinetics of calcite growth: surface processes and relationships to macroscopic rate laws. Geochimica et Cosmochimica Acta. 2000;64(13):2255-66.

Walderhaug $\mathrm{O}$. Kinetic modeling of quartz cementation and porosity loss in deeply buried sandstone reservoirs. AAPG Bull. 1996;80(5):731-45. 
Wendler F, Okamoto A, Blum P. Phase-field modeling of epitaxial growth of polycrystalline quartz veins in hydrothermal experiments. Geofluids. 2016;16(2):211-30

Westphal H, Eberli GP, Smith LB, Grammer GM, Kislak J. reservoir characterization of the Mississippian Madison Formation, Wind River basin, Wyoming. AAPG Bull. 2004;88(4):405-32.

Yi-Yeoun KA. A critical analysis of calcium carbonate mesocrystals. Nat Commun. 2014;5:4341.

Submit your manuscript to a SpringerOpen ${ }^{\circ}$ journal and benefit from:

- Convenient online submission

- Rigorous peer review

- Open access: articles freely available online

- High visibility within the field

- Retaining the copyright to your article

Submit your next manuscript at $\boldsymbol{\Delta}$ springeropen.com 